\title{
EL CONTEXTO HISTÓRICO DIDÁCTICO DE LA INSTITUCIONALIZACIÓN DE LA QUÍMICA COMO CIENCIA
}

\author{
Adriana Patricia Gallego Torres ${ }^{1}$, Rómulo Gallego Badillo² y Royman Pérez Miranda ${ }^{2}$ \\ adpgallegot@udistrital.edu.co rgallego@pedagógica.edu.co royman@pedagogica.edu.co
}

[Recibido en septiembre de 2008, aceptado en enero de 2009]

\section{RESUMEN $(\underline{\text { Inglés })}$}

Se dan a conocer algunas conclusiones derivadas de revisiones históricas, sobre cómo la química y la labor de los químicos fueron constituyendo paulatinamente esta ciencia, logrando su institucionalización y el reconocimiento social de su actividad de producción de conocimiento. Se entiende aquí la institucionalización en los términos que han establecido los historiadores sociales de las ciencias, destacándose la poca atención que han prestado a los procesos didácticos para este cometido. El punto de partida que se adopta, es el de que la conversión de las ciencias en ciencia escolar contribuyó significativamente a dicha institucionalización. Se examina el papel de los textos de enseñanza que formularon discípulos e incidieron en la creación y consolidación de una comunidad científica sin olvidar por supuesto, el rol cumplido por las revistas especializadas en química y la industrialización.

Palabras clave: Historia social de las ciencias; institucionalización; textos de enseñanza; revistas especializadas.

\section{INTRODUCCIÓN}

La pregunta de la cual se busca dar una respuesta admisible, es aquella que desde la historia social de las ciencias, indaga cómo se llevó a cabo la institucionalización de la química, para que el trabajo de los químicos fuera aceptado y valorado por la sociedad, por lo menos a partir del siglo XVII. No se ocupa este trabajo de un análisis de la historia interna (Laudan, 2005; Barona 1994) que condujo a la admisión en cada país de los diferentes modelos químicos que se fueron construyendo, desarrollando y modificando a lo largo de ese proceso de institucionalización para el cual se pretende elaborar una aproximación. En consecuencia, no se presenta una discusión en torno a las concepciones epistemológicas de los textos de enseñanza que han sido y son objeto de investigación por parte de otros especialista (Bertomeu y García, 2004; Bensaude - Vincent, Bertomeu y García, 2002).

\footnotetext{
${ }^{1}$ Profesora del Doctorado Interinstitucional en Educación. Universidad Distrital Francisco José de Caldas. Bogotá, D. C. Colombia.

${ }^{2}$ Profesores de la Universidad Pedagógica Nacional, Bogotá, D. E. Colombia.

Grupo de Investigación Representaciones y Conceptos Científicos - Grupo IREC
} 
El análisis, para reiterarlo pretende aproximarse a una mirada desde la sociología del conocimiento, en el supuesto de que la elaboración de textos de de enseñanza fue un emprendimiento indispensable por quienes persiguieron que sus concepciones y su actividad fuera reconocida socialmente en Inglaterra, Francia y Alemania. El estudio obligó a una fundamentación inicial acerca de la sociología del conocimiento científico. El Grupo de Investigación IREC venía ya discutiendo los orígenes de estas investigaciones, puntualizando que fueron los trabajos de R. K. Merton de 1938 "Science, technology, and society in seventeen century England" y de 1949 "Theory and social structure", con los que se inician las explicaciones de las condiciones culturales, políticas y económicas que hicieron posible en la Inglaterra del siglo XVII que versión de ciencia y de actividad científica que se inicia con Galileo y sus posteriores practicantes recibieran la estima y el reconocimiento social, esto es su institucionalización.

En dichas investigaciones se delimitan esas condiciones que puntualizan en las especiales relaciones que se establecieron entre ciencia, técnica y poder político militar. Indica Merton que antes de que se produjera ese proceso de institucionalización, es decir de la admisión de la ciencia como un valor cultural, las justificaciones que los miembros de la comunidad científica esgrimieron para justificar su actividad, estuvieron por fuera de las razones intrínsecas de su propio quehacer. El problema posiblemente no fue de la lógica interna de las explicaciones acera del funcionamiento del mundo, por que ya y desde siempre desde lo religioso existía. Fue así, por cuanto acudieron a los valores morales y utilitarios del puritanismo (Barona, 1994).

Estos estudios demuestran que el origen y desarrollo de la ciencia moderna se hallan estrechamente relacionados con el origen y desarrollo de la sociedad moderna, por lo que se encuentran ligados a la tecnología a los valores, intereses y estructuras básicas del capitalismo. Se institucionalizó como el modelo cognoscitivo de la industrialización. Llegó a ese mundo como un bien de consumo en íntima relación con los constructores de tecnología. Los historiadores sociales de la ciencia se han opuesto a las versiones que han instaurado el mito de una ciencia pura, derivada de actos mentales aislados, que se producen en la contemplación incontaminada, que se sustenta en la búsqueda del conocimiento por sí mismo, independiente de hechos sociales y de la cultura en la que en es producida. Ser agrega en contra, que si bien alguno científicos trabajan impulsados por nobles motivaciones, lo que producen sirve a intereses sociales (Restivo, 1992).

Los interrogantes que han dirigido estos estudios, son los que persiguen dar cuenta de cómo surgieron las ciencias de la naturaleza y se constituyeron como tradición; cómo se organizó la investigación; qué es lo que ha determinado los cambios en la organización científica; y, cómo se relacionan estos cambios con la investigación. Todos inscritos en el problema de qué es lo que ha hecho que el conocimiento científico se haya elevado como único entre las instituciones productoras de cultura, para elaborar una explicación admisible en torno a los orígenes de la ciencias, en el siglo XVII y su promoción en cuatro siglos a una posición de monopolio cognitivo sobre ciertas esferas de decisión (Vessuri, 1992). 
En las reconstrucciones histórico-epistemológicas de la química, de carácter predominantemente "internalista". Hay que destacar en estas reconstrucciones, primero y sin entrar en mayores consideraciones, el dominio que se instauró para juzgar aquello que aspirara a ser considerado como científico. El primer patrón establecido fue el que impuso I. Newton en la "Principia" y en la "Óptica". Los químicos, desde una no crítica aproximación epistemológica positivista y a partir de una mirada "fisicalista", historiaron y dieron cuenta del desarrollo de su ciencia, siguiendo este patrón. En el caso por ejemplo del libro G. Bachelard publicado 1953, la química desde esa perspectiva fisicalista (Bachelard, 1976).

Esta perspectiva seguirá siendo dominante, incluso en la nueva reconstrucción que propone B. W. Jensen (1998a; 1998b; 1998c), aun cuando parece acudir a la categoría kuhniana de "Revolución científica", (Kuhn, 1972). La categoría de "Revolución científica" ha sido objeto de críticas por parte de los historiadores de las ciencias (Bowler y Morus, 2005). Jensen elaboró una propuesta histórica innovadora, en relación con las habituales versiones positivistas (Lockemann, 1960; Brock, 1998; Ihde, 1984). Para dar cuenta del proceso de construcción de esta ciencia, acude a tres dimensiones (composición y estructura, energética y temporal) y tres niveles (molar, molecular y electrónico) (Jensen, 1998a).

La construcción histórica de la química puede también ser caracterizado por la delimitación y diversificación paulatina de su objeto de conocimiento inicial (Bachelard, 1979) con las correspondientes precisiones instrumentales. Habría entonces que tener en cuenta en esas reconstrucciones racionales la formulación, desarrollo, modificación y sustitución del modelo científico para el átomo de J. Dalton (Gallego Badillo, Pérez Miranda, Gallego Torres y Torres de Gallego, 2006) y de los que le sucedieron.

La segunda, "externalista" deviene del interés de quienes desearon que su actividad fuera reconocida y se institucionalizara como científica. Este proceso tuvo como objetivo prioritario, la constitución de una comunidad de especialistas que trabajara para obtener esa valoración social y recibiera el apoyo financiero, con miras al sostenimiento de su actividad de producción de saber. Una de las metas fue la de convencer poco a poco a las nuevas generaciones de la importancia epistemológica, social y técnica de los modelos científicos que se elaboran. La intencionalidad fue tener discípulos, para que se vincularan, hicieran el relevo generacional y continuaran el proceso. Por tanto, una primera labor en este sentido fue la redacción y publicación de textos para la enseñanza y la divulgación. Una tercera, sería la creación de revistas especializadas. S. Toulmin (1977), dirá que el desarrollo científico es la obra de una genealogía de maestros y discípulos. Son estas últimas el objetivo principal de este estudio.

\section{LOS LIBROS DE ENSEÑANZA EN LA HISTORIA DE LA INSTITUCIONALIZACIÓN DE LA QUÍMICA}

Con la publicación de la "Iatroquímica" de Paracelso (1493 - 1541), a mediado de la primera mitad del siglo XVI aproximadamente. El objetivo fue posiblemente, convencer y crear simpatizantes que dieran a conocer sus ideas acerca de la influencia de los preparados químicos en la cura de enfermedades. Destáquese que Paracelso fue profesor de medicina en 1526, en la Universidad de Basilea, de la que fue 
expulsado en razón de su desdén por las ideas tradicionales sobre las prácticas médicas.

Le sigue Jean Beguin (1550 - 1620), quien en 1610 escribe para sus estudiantes "Tyrocinium chymicum"; texto este que es considerado como el primero en ocuparse del problema de la enseñanza de las nociones químicas de ese tiempo. Es el siglo XVII, en el que I. Newton (1642 - 1727) da a conocer su "Principia", y es en el que se funda la "Royal Society" y la primera revista especializada, la "Phylosophical Transaction" en 1665, con la que se inicia la tradición de publicar los aportes en este nuevo medio de circulación comunitaria. Habría que inscribir en este listado de textos de enseñanza, "The Sceptical Chymist", que dio a conocer en 1661, Robert Boyle (1627 - 1691), como una crítica radical a las ideas alquimistas, para hacer de la química una rama de la filosofía natural.

En 1667 Johann Becher (1635 - ¿1682?) pone a circular su "Physica subterranae", en el que propone la existencia de tres "tierras" entre ellas la "tierra grasa" o materia combustible. Esta será el argumento central de George Stahl (1660 - 1734) para que en 1697, en su "Zymotechnia fundamentalis", para proponer su modelo del flogisto; modelo que será el primero propiamente químico, dada su sistemática y consistencia conceptual y metodológica (Izquierdo, 1983). Fue este el modelo del que dispusieron en su tiempo químico destacados como C. W. Scheele (1742 - 1786), J. Priestley (1733 - 1804) y H. Cavendish (1731 - 1810), entre otros (Laín Entralgo y López Piñero, 1963).

A este proceso de institucionalización didáctica se vinculará William Lewis (1708 1781) cuando publica en 1746 su "Course of practical chemistry" y, en 1758, "Laboratory laid open", dentro del modelo del flogisto. Para estos años la química es asumida aún en términos de una práctica meramente artesanal, derivada de las elaboraciones de los artesanos metalurgistas: La química más que ciencia es una práctica profesional que ha de aprenderse en largas y continuadas horas de entrenamiento en los laboratorios. En 1675, Nicolás Lémery (1645 - 1715) escribe su "Cours de chimie" que entró a ser parte de esa tradición en la que la química de entonces era tan solo una "disciplina escolar" cuyos practicantes perseguían y luchaban porque su ocupación intelectual fuera admitida socialmente. Si se repara en el título del libro de Lewis de 1746 y el de Lémery, esta ciencia ya posee un nombre que la identificará y diferenciará.

El cambio de modelo vendrá con la revolución científica iniciada por A, L. Lavoisier (1743 - 1794), al demostrar con la balanza que el modelo del flogisto era insostenible. Para que esa revolución por él creada perdurara, redactó y dio a la publicidad en 1789, su "Traité elementaire du chemie", dedicado a las nuevas generaciones interesadas por estos problemas y no a los químicos en ejercicio que seguirían enseñando las ideas flogísticas dominantes. A partir de 1801 el "Tratado" se convirtió en un modelo de texto para la enseñanza de la química. En esta perspectiva hay igualmente que relacionar el texto de A. E. Fourcroy (1737 - 1816) de 1792, "Philosophie chimique", que con el de Lavoisier constituyera una guía para la enseñanza de esta ciencia durante varias décadas. 
El año de 1808 es relievante, por cuanto John Dalton (1776 - 1844) propone el primer modelo químico icónico sobre el átomo, en su "A new system of chemical philosophy", dedicada a los alumnos y profesores de las universidades de Edimburgo y Glasgow. Dos años antes, en 1806, J. J. Berzelius (1779 - 1848) escribiría para sus estudiantes de medicina Djurkemi (Química animal). Entre 1808 y 1830, también con propósitos de enseñanza, daría a conocer en seis tomos su "Lärbok i organisk kemien" (Lockemann, 1960), en donde admite el modelo atómico de Dalton y las leyes de la composición definida y de las proporciones múltiples. La actividad de los químicos va adquiriendo progresivamente su estatuto científico; estatuto dentro del cual se formarán las nuevas generaciones de estudiantes interesados en esta actividad de producción de conocimiento.

En 1850, Charles Gerhardt (1816 - 1856) publica uno de los textos también significativo para la enseñanza de la química, su "Traité de chimie organique", en el que realiza una sistematización de la química orgánica que dio pie para que Dimitri Mendeléiv (1834 - 1902) escribiera su "Principios de química", en 1867, al parecer con dos objetivos; involucrar a sus estudiantes de la universidad de San Petersburgo en las discusiones que se dieron en el Congreso de Karlsruhe y aplicar a la química inorgánica la sistemática en química orgánica que Gerhardt había elaborado (Lockemann, 1960; Brock, 1998).

Stanislao Canizzaro (1826 - 1910), en 1858 publica su texto "Sunto di un corso di filisofia chimica", en el que reconocía y argumentaba su admisión del átomo químico, con base en la hipótesis de Amadeo Avogadro (1776 - 1856). En este texto, que distribuyó ampliamente en el Primer Congreso Internacional de Química, celebrado en la ciudad alemana Karlsruhe en 1860. Frente a las discusiones que suscitó la hipótesis, Cannizaro en este Congreso sugirió que dados los desacuerdos, habría necesariamente que abandonarla (Lockemann, 1960). No obstante, tanto el texto de enseñanza de Gerhardt como el de Canizzaro se convirtieron en las nuevas versiones didácticas en las que se formaron las nuevas generaciones de químicos en la segunda mitad del siglo XIX. La actividad de los químicos se había ya institucionalizado.

Agréguese a la lista de textos, que C. R. Fresenius (1818 - 1897) en la segunda mitad del siglo XIX redacto textos didácticos sobre el análisis químico cualitativo y cuantitativo. F. Wöhler escribió un "Manual de química", cuya primera parte la dedicó a la inorgánica, publicada en 1831, y la segunda a la orgánica, en 1840. L. Gatermann (1860 - 1920) publicó en 1896 su manual de "Practicas de química orgánica". En los comienzos del 1800, W. Lewis dio a conocer su "Course of practical chemistry" Dominan las investigaciones en química orgánica. Finaliza el siglo XIX.

\section{INICIACION DE LA FORMACIÓN ACADEMICA DE LOS QUÍMICOS}

El caso de la química es ejemplo de que inicialmente, las concepciones y las prácticas de sus representantes no fueron consideradas como una rama de la filosofía natural. Esta apreciación fue formulada en el siglo XVIII, por I. Kant (1724 - 1804), tomando como base la dinámica newtoniana y la geometría de Euclides. Afirmó que carecía de los fundamentos matemáticos para asumirla como ciencia $y$, por tanto, había que considerarla tan solo un "arte sistemática" (Kant, 1987). A finales de este siglo y comienzos del XIX, se dice que no se necesitaban conocimientos matemáticos 
complejos para comprender las aportes de A. L. Lavoisier (1743 - 1794), de J. Dalton $(1766$ - 1844) y de J. L. Gay-Lusaac (1787 - 1850) (Schneer, 1975).

La matemática compleja se introducirá en la química con la formulación de la fisicoquímica a finales de ese siglo XIX. Su enseñanza se encontró con el obstáculo de que la matemática que dominaban los químicos les impedía acceder a las nuevas explicaciones basadas en la termodinámica clásica. Los químicos orgánicos alemanes desde su tradición experimentalista, no recibieron con agrado a la fisicoquímica, por lo que W. Ostwald (1853 - 1932), escribió un texto para la enseñanza de esta nueva disciplina, que fue traducido al inglés (Brock, 1998). Ostwald es el fundador con J. H. van't Hoff (1852 - 1911) y S. Arrhenius (1859 - 1927) de esta nueva disciplina química. Para la consolidación de este espacio de producción de conocimiento, crearon la revista "Zeitschrift für phisikalische Chemie", que abrió el espacio indispensable para una matematización de las explicaciones sobre los problemas energéticos implicados en los procesos químicos.

En cuanto a la enseñanza, antes del siglo XIX quienes se interesaban por los "problemas químicos" eran los médicos; médicos que a su vez, contribuyeron a elevar a la categoría de práctica profesional el saber de los "boticarios" o "practicantes de farmacia", en cuyo seno se formaron inicialmente los "químicos". Habrá que esperar hasta 1805 cuando Friedrich Stromeyer (1776 - 1835) creara un programa de formación basado en las prácticas de laboratorio, es decir, una enseñanza práctica de esta ciencia. Luego, en la tercera década de ese mismo siglo, hacia 1825 Justus von Liebig (1803 - 1873), siendo aún joven, en la ciudad alemana de Giessen formula y pone en práctica un plan de estudios con la intención de que los estudiantes se sumergieran en un aprendizaje de la química, desde el punto de vista experimental e investigativo. Hay que recordar que Jöns Jacob Berzelius (1779 - 1848) fue nombrado profesor en 1807 y en su Academia-Laboratorio egresaron reconocidos investigadores alemanes como C. G. Gmelin (1792 - 1860), H. Rose (1795 - 1864), G. Rose (1798 1873), F. Wöhler (1800 - 1882) y G. Magnus (1802 - 1870) (Lockemann, 1960).

Liebig además, desde 1832 editó los "Annalen der Pharmazie", que en 1840 con la colaboración de F. Wöhler, convirtió en los "Annalen der Chemie und Pharmazie"; una revista que aparece hoy con el nombre de "Anales de Liebig". Además, en 1842, Liebig edita el "Diccionario de Química pura y aplicada". Sobre la formación de Liebig habría que decir que en un principio acudió al profesor K. W. Kastner $(1783$ - 1857) de la Universidad de Bonn y Erlangen. Kastner prometió enseñarle los secretos de la marcha analítica de cuyo aprendizaje terminó decepcionado. En 1823 viajó a Paris y para asistir a las conferencias de J. J. Gay-Lussac (1787 - 1850), J. J. Thenard (1977 - 1857), P. S. de Laplace (1749 - 1827), G. de Cuvier (1769 - 1832) y otros investigadores. Gay-Lussac lo admite en su laboratorio como colaborador (Lockemann, 1960).

La extensión de la enseñanza sistemática de la química alemana se llevó a cabo primero en Gran Bretaña y después en los Estados Unidos, países estos en los que se siguió en sus inicios la concepción de J. Liebig. En la década del sesenta del siglo XIX, en Gran Bretaña esa extensión se debió a A. W. Hofmann (1918 - 1892), discípulo de Liebig; a E. Frankland (1825 - 1899); a John Tyndall (1820 - 1893), ambos alumnos de R. G. Bunsen (1811 - 1889); y, a Henry Edward Armstrong (1848 - 1937), quien 
se doctoró con Herman Kolbe (1818 - 1884). Hofmann abogó por una enseñanza en la que se compartiera con los estudiantes los conceptos y el lenguaje conceptual y metodológico comunitario basado en la hipótesis de Avogadro, en cuanto la distinción entre átomos y moléculas y en el concepto de valencia.

Después de un análisis crítico de la enseñanza de la química en el Reino Unido, en una intervención ante el Royal College of Chemistry de Londres, en 1864, Hofmann propone una reforma en la que la enseñanza de esta ciencia debería llevarse a los niveles elementales de la educación (Brock, 1998). En este mismo año Hofmann se traslada a Alemania, donde ejerce como profesor en la Universidad de Bonn y a partir de 1865, en la de Berlín. En 1867 funda la "Deutschen Chemischen Gesellschaft" (Lockemann, 1960). La química estaba ya vinculada a los procesos de industriales, lo que contribuyó definitivamente a su institucionalización social.

E. Frankland por su parte, inicia su labor como profesor de escuela entre 1847 y 1848 ; tan solo había tenido una iniciación técnica en química como empleado de un laboratorio farmacéutico; formación que completó con las lecciones que le impartió J. Tyndall, físico e igualmente maestro de escuela. Después de doctorarse con Bunsen, Frankland retorma la enseñanza en 1851. Sucede a Hofmann y se convierte en el principal profesor del "Department of Sceience and Art" (DSA). A partir de 1865 fue el responsable del establecimiento de los planes de estudio para la enseñanza de la química y de los exámenes de los jóvenes estudiantes de esta ciencia. Entre 1870 y 1880, diseñó y lideró una campaña para dotar de laboratorios a todas las clases de ciencias.

Es dentro de esta gestión que le preocupa la formación de profesores de química, ya que tales laboratorios no serían útiles si los profesores no eran competentes para realizar con ellos clases prácticas. Así, en julio de 1870 prepara un curso para que observaran su concepción del papel del laboratorio en la enseñanza de la química al que invitó a sesenta y cinco profesores del DSA y de toda Inglaterra. Parecería entonces que fue Frankland, dentro de su versión de química y de su enseñanza, el primero en preocuparse por la "formación" de profesores en esta ciencia. Frankland publica en 1866 su "Lecture notes for chemical students". Este texto brindó oportunidad a las nuevas generaciones de químicos, para comunicarse empleando fórmulas gráficas y estructurales. Además, el DSA le editó el "folleto" "How to teach chemistry", en el que recogió las seis clases matinales de práctica que desarrolló en su curso para la preparación de profesores. Recogía un total de 109 experimentos que, para Frankland, todo profesor de química debía realizar frente a sus estudiantes. (Brock, 1998).

Le sucedió en la tarea de imponer en Gran Bretaña el esquema de Liebig, H. E. Armstrong (1848 - 1937). A su regreso de Alemania en 1870, pensando en que los estudiantes de química habían sido "completamente embrutecidos" por el autoritarismo de los profesores y el seguimiento al pie de la letra de los textos de enseñanza, que no se ocupaban de la elaboración de explicaciones químicas de problemas propios de la vida diaria y que eran incapaces de pensar autónomamente, pues memorizaban datos y fórmulas para superar los exámenes. Si bien sus antecesores habían logrado introducir la enseñanza de esta ciencia en las escuelas, 
facultades y clases nocturnas, no se habían lideradoen torno a cómo se debían enseñar las ciencias o cómo elaborar un plan de estudios para ellos (Brock, 1998).

Armstrong es el primero en aproximarse a una concepción epistemológica positivista sobre la enseñanza de la química, ya que en su propuesta abogó por la introducción del "Método Científico". Según él, se debía propender por proceso de autodidaxis a las que llamó "heurismo", en la perspectiva de una enseñanza por descubrimiento dirigido. Fue partidario de la idea de que los estudiantes no olvidaban aquello que ellos descubrían por sí mismos y que esa autodidaxis les posibilitaba interesarse e involucrarse en la búsqueda de soluciones a los problemas que esa enseñanza debería plantearles. Sugirió que la química debía aprenderse de manera gradual, partiendo de lo conocido a lo desconocido. Para ello propuso una serie continua y de creciente complejidad en las prácticas de laboratorio (Brock, 1998).

H. E. Armstrong, en 1884, se llevó a cabo la Conferencia Internacional de Educación, en el "City and Guilds of London Institut" en South Kensington. Se afirma que en el desarrollo de esta, conferencia el 5 de Agosto de ese año, Armstrong presentó su visión crítica de la versión de enseñanza de las ciencias dominante, en particular porque se centraba en la actividad del profesor y no en el aprendizaje del alumno que debía realizarse a través de la experiencia y la experimentación.

\section{EL PASO DE LOS TEXTOS DE ENSEÑANZA A LAS REVISTAS ESPECIALIZADAS}

Ya se ha mencionado la labor de Liebig en relación con la creación de la primera revista especializada propiamente química. Siguieron otras en las que se pusieron en circulación en la comunidad las nuevas propuestas. Con un problema epistemológico, conservar el esquema de los químicos orgánicos alemanes, cuyos éxitos innegables se fundaban en la síntesis de los azucares naturales, la síntesis del primer edulcorante y el primer fármaco de diseño contra la sífilis, el "Salvarsan" (Gallego Badillo, Pérez Miranda, Gallego Torres y Torres de Gallego, 2006).

Estos órganos especializados de comunicación entre los químicos surgen a finales del siglo XVIII por iniciativas particulares. Las revistas de mayor reconocimiento, entre alemanes y franceses, fueron por ejemplo, la "Crell's Chemissches Annalen" de 1778 y la fundada por Lavoisier, Berthollet y Fourcroy, "Annales de Chemie" de 1789, que se constituyó en la principal revista gala de química; la crearon debido a que los editores del "Journal de physique, de chemie et d'histoire naturelle", se negaron a publicar las comunicaciones en contra del modelo del flogisto y en razón de que hubo impedimentos para editar la versión francesa del "Crell's Chemissches Annalen". En 1815 los "Annales" cambiaron a "Annales de Chimie et Physique" que se escindió en 1914 en "Annales de Chimie" y "Annales de Physique" (Leidler, 1995).

En Italia y también por iniciativa privada, se funda en 1790, "Annali di Chimica". Alemania es la cuna de estas revistas especializadas, ya que en 1778 , ven la luz "Chemisches Annalen", abierta a las concepciones del modelo del flogisto y cuya periodicidad terminó en 1804, cuando se inicia el abandono de este modelo. La importancia de los Annalen pasó a ser ocupada por la "Allgemeines Zeitschrift für Chemie", que se hizo vocera del modelo de Lavoisier. En 1834 cambió el nombre por el de "Zeitschrift für praktische Chemie", que bajo la dirección de Kolbe, se especializó 
en los desarrollos de la química orgánica, aún cuando abrió sus páginas a artículos procedentes de la naciente fisicoquímica (Brock, 1998).

En Alemania diferentes revistas fueron fundadas en los inicios del siglo XIX, siendo la más reconocida "Annalen der Pharmacie" de 1832, en razón de que los aspectos farmacéuticos de la química eran los que mayor atención recibían por parte de los especialistas, era una revista no institucional. De las revistas alemanas es menester mencionar también la "Journal für praktische Chemie" que nació en Laipzig en 1834 y el "Berrichte der deutschen chemischer Gesellschaft", que comenzó en Berlín en 1868. Agréguese que es la creación de los programas académicos para la formación profesional de químicos, los que van a potenciar la proliferación de esta revistas especializadas y la utilización de las mismas en la enseñanza.

\section{LAS SOCIEDADES QUÍMICAS}

Los estudios acerca del trabajo de A. L. Lavoisier hablan en favor de que creó a su alrededor un grupo de investigación multidisciplinario que demuestra que la "Revolución científica" que él creo en química (Bensaude- Vincent,1991), no fue obra de un genio aislado, como se suele presentar en las lecciones que tratan del papel por él desempeñado; algo que es indicativo de que los químicos habían ya admitido su condición de una comunidad de especialistas.

Esta referencia es un punto para reafirmar que las relaciones anteriores sobre la institucionalización de las actividades de los químicos, sería incompleta si no se soporta en la creación de las "Sociedades de Químicos", que a diferencia de los practicantes de la física, por ejemplo, se transformaron en organizaciones de defensa de la profesión. Se puntualiza en que el inicio de la admisión institucional de la química se produce a partir de la mitad del siglo XIX, ya que en 1841 se crea "The Chemical Society of London" que recibió carta real de reconocimiento en 1848, y cuyo primer presidente fue T. Graham. Esta asociación fundó en 1858 las "Memoirs of the Chemical Society", cuyo nombre se cambió en 1862, por el de "Journal of the Chemical Society" (Leidler, 1995).

Esta asociación cuyo primer objetivo parece que fue la de intervenir a favor de que las industrias contrataran a profesionales químicos egresados de los programas académicos ya establecidos, en oposición a que esas industrias acudían en gran número a los tecnólogos químicos. Frente a esta situación los tecnólogos conformaron en 1881 la "Society of Chemical Industry" en la que en 1882, editaron su propio "Journal", que se adelantó a la alemana "Verrein Deutscher Chemiker", de 1887, "Zeitschrift für angewandte Chemie", la que hoy se denomina "Angewandte Chemie". En lo tocante a los ingleses "The Chemical Society of London" se constituyó en el modelo para la organización de sociedades químicas análogas en todo el mundo. Estos hechos muestran que la constitución de la comunidad de especialistas en química siguió caminos distintos de la de los físicos.

En 1858 se crea la "Societé Chimique de Paris", que en 1907 cambió a "Societé Chimique de France". En el mismo año de su fundación editó "Bulletin de la Societé Chimique de Paris", que como era de esperarse cambió el título a partir de 1907 a "Societé chimique de France". En Alemania, la "Deutsche Chemische Gesellschaft", de 
1867, publicó su "Berichte", que rápidamente adquirió gran importancia. En Estados Unidos se organizó la "The American Chemical Society" en 1876, que tres años después lanzó su "American Chemical Journal". En 1879, I. Remsen (1848 - 1927) empezó a editar "American Chemical Journal" como competencia a la revista de la Asociación estadounidense. En 1914 la "American Chemical Journal" fue absorbida por la "Journal of the American Chemical Society", siendo editor W. A. Noyes (1857 1941): entre 1902 y 1917, fue quien a su vez editó "Chemical Abstract" y "Chemical Revierps".

En la mayoría de los países industrializados se crearon a finales del siglo XIX estas asociaciones de químicos, las cuales cumplieron un doble papel, velar por el reconocimiento profesional de los químicos graduados en programas universitarios de pregrado y posgrado, con cierto tinte "sindical", y académico, por cuanto impulsaron la investigación química, al mismo tiempo que la organización de congresos y otras formas comunitarias de socialización de los resultados de esas investigaciones. Tales asociaciones no pueden ser asimiladas a la categoría de academias de ciencias, a las que pertenecieron muchos de los químicos reconocidos en su tiempo.

Para cerrar este subtítulo es preciso mencionar otras revistas significativas, por fuera de la intención de elaborar una lista exhaustiva de ellas. Así, en 1862, "Zeitschrift für analytischen Chemie"; la "Zeitschrift für Chemie", desde 1858 hasta 1871; en 1887, Ostwald y Van't Hoff, crean la "Zeitschrift für physikalische Chemie"; en 1892, "Zeitschrift für anorganische Chemie". En otras palabras, la actividad de los químicos comenzó a circular a través de los mismos medios de comunicación especializados, cuya praxis venía ya desde el siglo XVII. Acudiendo a la cienciometría, se hizo definitivamente ciencia y los químicos adquirieron el estatuto de científicos justamente porque sus elaboraciones fueron admitidas en estas revistas y citados por otros miembros de la comunidad en revistas del mismo estatutus.

La química se institucionalizó y el trabajo de producción de conocimiento de los químicos, con sus consecuencias industriales, recibió el reconocimiento por el que venían luchando desde el siglo XVII. Una comprensión de este hecho histórico requiere de un análisis en el que se relacionen los diferentes factores implicados: La conversión escolar de los modelos químicos y de la aproximación epistemológica desde la cual se enfrentó esa necesidad de socialización; el dominio de la versión experimentalista iniciada por Liebig; la creación de las asociaciones de químicos que adoptaron esta versión y su lucha por el reconocimiento profesional, en oposición a los farmaceutas y los tecnólogos en el contexto del desarrollo industrial; la paulatina construcción de una comunidad científica, en los términos de T. S. Kuhn (1972); la aparente adopción de la aproximación epistemológica empirista primero, y la positivista después, desde las cuales esa comunidad de químicos dejó de lado la pregunta por los presupuestos histórico-epistemológicos de los fundamentos conceptuales y metodológico de su objeto de conocimiento.

\section{eL CASO DEL Siglo VeINTE}

Reitérese que la versión de química y su enseñanza que se expande a partir de la segunda mitad del siglo XIX hasta bien entrada la segunda mitad del XX, es la experimentalista de carácter empirista primero y positivista después, basada 
principalmente en los desarrollos alcanzados por los químicos orgánicos. Además, es factible suponer que el paradigma dominante es el artesanal, de que para enseñar química basta con conocerla. Es durante este período que esta disciplina logra un reconocimiento indiscutible como ciencia (Gallego Badillo, Pérez Miranda, Gallego Torres y Torres de Gallego, 2007).

Para algunos especialistas la enseñanza de las ciencias de la naturaleza recibe especial atención con un hecho significativo: En 1957 ocurre el lanzamiento al espacio del primer "Sputnik" por parte de la otrora Unión Soviética. Este hecho despertó una conmoción política que puso en alerta y movilizó a las potencias occidentales, principalmente a la Gran Bretaña y a los Estados Unidos discutir las razones por las cuales la URSS había tomado ventaja en la carrera espacial. Una de las conclusiones a las que se llegó centró su análisis de los contenidos curriculares de ciencias que se hacían objeto de enseñanza en la educación secundaria. Se convocó entonces a las academias de ciencias y a las asociaciones de profesores de ciencias, con el fin de que propusieran soluciones.

Así, en los Estados Unidos se creó un primer proyecto dirigido a los institutos que se llamó "Chemical bond" que se puso en marcha en 1959; luego de su aplicación en las aulas y de las modificaciones sugerida por los resultados obtenidos, el equipo responsable redactó en 1964 un texto de enseñanza que fue de poco uso "Chemical system". Este texto se acompañó de un manual de laboratorio: "Investigating Chemical system". La concepción experimentalista - positivista heredada, imperó. El fracaso de este primer proyecto puede deducirse del surgimiento de otros. Uno de ellos, bajo la premisa de ser un proyecto de renovación de la enseñanza de la química para entusiasmar a las nuevas generaciones hacia el estudio y la investigación en esta ciencia, fue para el mismo año de 1964, el "Chemical Bond Approach", financiado por la "National Science Foundation"; proyecto este frente al cual la "American Chemical Society" (ACS) tuvo serios reparos, además de que los profesores de enseñanza media manifestaron abiertamente que ellos no habían sido consultados.

A la par que el proyecto "Chemical bond", para no quedarse por fuera del protagonismo, la ACS, también en 1959, financió la elaboración del denominado "CHEM study" o "The Chemical Education Material Study". El "CHEM study", como material de enseñanza estuvo conformado por una "Guía de laboratorio", una "Guía para el profesor" y u texto para los estudiantes. Los análisis de este material de enseñanza, permite afirmar que quienes participaron en su elaboración, de manera implícita pusieron en circulación sus concepciones experimentalistas y positivistas acerca de la química y su enseñanza. Hubo cierto reconocimiento a la historia del desarrollo de esta ciencia, dentro de las concepciones señaladas, por cuanto este material introducía a los profesores y estudiantes en el estudio de las leyes empíricas de los gases y no con la definición de átomo.

La inversión en la elaboración del "CHEM Study", se intentó recuperar con la traducción de este material a varios idiomas y culturas que no tenían una tradición de producción de conocimiento y una supuesta enseñanza de la química de conformidad con los cánones históricos. Esa estrategia comercial de recuperación incluyó también, el otorgamiento de becas para que, en el caso latinoamericano, los profesores de química recibieran un entrenamiento para su uso e implementación en sus respectivos 
países de origen. Por supuesto que ese entrenamiento estuvo principalmente dirigido a los profesores latinoamericanos que formaban profesores de química, en este caso. Se vendió el proyecto en un espacio social, cultural, político y económico en el que los fundamentos histórico-epistemológicos acerca de la construcción de una explicación científica de los acontecimientos del mundo natural, seguía atada a lo mitológico y religioso. A partir de la ignorancia de la era pospositivista que se inicia en 1935, con la publicación del libro de Popper, "Logik der Forschung" el "CHEM Study" socializó en la enseñanza que el método científico era el camino expedito para que los estudiantes de educación media comprendieran, en principio, la construcción de la química como ciencia y decidieran hacerse practicantes de esta ciencia.

Todos los programas anteriores acerca de la enseñanza de la química con todas las inversiones institucionales, en términos de resultados la ACS, fracasó en su intención de una enseñanza de la química que aumentara el número de norteamericanos interesados en estudiar y de producir ciencias. Se les ha denominado genéricamente los "programas alfabeto" (Novak, 1982). Se elaboraron a partir de una mirada acerca del estatuto científico de las ciencias de carácter empiropositivista, centrada en la enseñanza por descubrimiento dirigido. Desde esta perspectiva tales programas alfabeto no fracasaron en sus propósitos por interesar a las nuevas generaciones en el estudio de las ciencias, incluida la química, pues presentaron una versión de ciencia "terminal", la basada en técnicas de laboratorio estandarizadas, por fuera de la aventura histórica de la construcción no lineal del conocimiento científico en general, y del químico en particular. Una química "terminal" sin la historia de su construcción, en la que predomina la idea de un producto para "consumo" de la población estudiantil, vía el ejercicio de una ocupación eminentemente tecnicista.

Se siguen escribiendo libros de texto para la enseñanza de la química, tanto universitaria como secundaria. Los textos investigados por el Grupo IREC investigación muestran que siguen presentando una química "producto" y no como una actividad que históricamente fue adelantada por colectivos de especialistas; descontextualizada, es decir, sin una referencia a los distintos contextos culturales en que esta ciencia, por los esfuerzos de esos colectivos, adquirió su reconocimiento social (Camacho González, Gallego Badillo y Pérez Miranda, 2007).

Esa versión de química que socializan los textos de enseñanza, si bien imponen una reconstrucción histórica de carácter positivista, en sus primeros capítulos y dentro de la mirada "fisicalista" suelen comenzar con el estudio de los modelos atómicos, dejando la convicción de que la historia de esta ciencia comienza con J. J. Thomson, E. Rutherford, N. Bohr y la mecánica cuántica. El modelo atómico de J. Dalton, cuando es mencionado se suele aludir a él como un momento remoto sin importancia. Esa discusión que predominó hasta finales del siglo XIX entre atomistas y equivalentistas, brilla por su ausencia y el hecho histórico de que la admisión de la hipótesis atómica no fue fácil. Además, en la transposición didáctica (Chevallard, 1985) del modelo de Rutherford (Cuellar Fernández, Gallego Badillo y Pérez Miranda, 2008), son muchas las tergiversaciones que introducen estos textos.

Posiblemente transcurra aún mucho tiempo antes de que la reconstrucción histórica que propone W. B. Jensen (1998a; 1998b; 1998c) entre a ser considerada como una versión a partir de la cual socializar una nueva perspectiva para la enseñanza de la 
química en todos los niveles de los sistemas educativos. En la medida en que la enseñanza de la química se apoye en la historia del la construcción de esta ciencia, la esperanza, de que la construcción de actitudes positivas hacia ella podría mejorar.

\section{LA CONSTRUCCIÓN DE ACTITUDES POSITIVAS HACIA LA QUÍMICA}

Ya C. B. Faust (1989) al sostener que la moderna enseñanza de la química puede incluir trabajos prácticos de un tipo distinto de los de la física y de la biología y que el método científico puede ser fomentado en cada una de estas ciencias, para el caso de la química afirma que esos trabajos prácticos deben partir del convencimiento de que el diseño e interpretación de las investigaciones en esta ciencia son frecuentemente únicos, propias de ella, por lo que debe ser trabajada por separado en los currículos escolares.

Un estudio particularmente significativo realizado en Bélgica por D. Neerinck y C. R. Palmer (1979) entre estudiantes que habían elegido la química como campo de especialización en su último año de estudio, a quienes se les presentaron las opciones de química orgánica, fisicoquímica, química inorgánica, bioquímica y química analítica, empleando un instrumento basado en el "Diferencial semántico de Osgood", los resultados obtenidos entre los estudiante que aún no habían elegido el curso de especialización, fueron: El 35\% mostró preferencias por la química orgánica y el $61 \%$ por la bioquímica. Para estos investigadores tales resultados no fueron sorprendentes, dado que las referencias bibliográficas a las que acudieron hablaban en favor de que los estudiantes no elaboran actitudes negativas hacia la química orgánica, mientras que sí la manifestaban con respecto a la fisicoquímica y a la química inorgánica.

Estos resultados no pueden ser interpretados en términos de aptitudes o de nacer genéticamente programados para entender los problemas asociados con los modelos de las diferentes disciplinas de la ciencia química. A manera de hipótesis habría que afirmar que tiene necesariamente que ver con la versión de esta ciencia que se hace objeto de trabajo en el aula. En ella, por supuesto, habría que incluir los textos de enseñanza mediante los cuales los profesores de química aproximan al estudiantado a la lógica histórico-epistemológica a través de la cual, de una manera no lineal se construyó esta ciencia (Stengers, 1989).

\section{A MANERA DE CONCLUSIÓN}

Hay que resaltar que tanto los historiadores "internalistas" como los "externalistas" no han hecho objeto de estudio el papel cumplido por la educación en ciencias y los textos de enseñanza en la institucionalización social de cada una de las ciencias de la naturaleza. Los externalistas principalmente, deberían ser aquellos de quienes esperar este tipo de trabajos. En la literatura revisada por los responsables del presente artículo no encontraron referencias específicas al respecto. Se piensa que se ha demostrado en el caso de la química, que tales textos de enseñanza contribuyeron a ese cometido.

Aun cuando se reconoce que las referencias bibliográficas de apoyo son insuficientes para apoyar la hipótesis de que en ese proceso de institucionalización desempeñaron un papel trascendental la escritura de textos de enseñanza, podría ser admisible que 
en una sociedad determinada ese proceso de institucionalización requiere necesariamente de convertir cada ciencia naciente en objeto de enseñanza. De hecho hay otras estrategias también significativas como la transformación de los logros científicos en el mejoramiento de los sistemas de producción de mercancías, sobre lo cual se ha soportado el desarrollo científico - tecnológico a partir del siglo XVII.

En consecuencia se sugiere que con esta contribución, se abre la posibilidad de construir un campo de investigación que podría titularse "Historia social de la didáctica de las ciencias o de la educación en ciencias". En este sentido pretende ser un aporte inicial. Por lo general, la enseñanza de una ciencia descontextualizada y ahistórica no somete a discusión los procesos que desarrollaron en un principio los colectivos de especialistas, para que su actividad fuese aceptada socialmente y por tanto, institucionalizada.

El grupo de investigación que firma la presente contribución adelanto un proyecto de investigación aprobado y financiado por la Universidad Pedagógica Nacional, sobre la creación, desarrollo, persecución y desaparición de la Escuela Normal Superior de Colombia (ENSC), fundada en 1933 dentro de un proyecto de educación pública y laica para todos, suprimida en 1952. La ENSC, una institución para la formación de profesores de ciencias, fue la primera en el país en que esas ciencias se enseñaron "per se", no asociadas a la formación de médicos e ingenieros. En el mercado bibliográfico nacional no había textos didácticos disponibles, y fueron los egresados de la ENSC quienes se dedicaron a escribirlos; incluso quienes se reconocen como practicantes profesionales de lasa ciencias a lo largo del siglo XIX, no se ocuparon de dicha tarea. Los intentos por una institucionalización de la actividad científica en Colombia, se aplazará hasta la década del setenta del siglo XX (Gallego Badillo, Pérez Miranda y Rincón Pabón, 2008).

\section{REFERENCIAS BIBLIOGRÁFICAS}

Barona, J. L. (1994). Ciencia e historia. Debates y tendencias en la historiografía de la ciencia. Valencia: Guada.

Bachelard, G. (1976). El materialismo racional. Buenos Aires: Paidos.

Bachelard, G. (1979). El racionalismo aplicado. Buenos Aires: Paidos.

Bensaude - Vincent, B. (1991). Lavoisier: una revolución científica. En: Historia de las ciencias, M. Serres (Ed.), pp, 410 - 435. Madrid: Cátedra.

Bensaude - Vincent, B., Bertomeu, J. R. y García, A. (2002). Looking for an order of things: Textbooks and chemical classifications in nineteenth century France. Ambix, 49(3), 227 - 251.

Bertomeu, J. R. y García, A. (2004). Atoms in French chemistry textbooks during the first half of the nineteenth Century: The Eléments de chimie médicale by Mateu Orfila. Nuncius. Journal of the History of Science, Vol. 19(1), $77-119$.

Bowler, J. P. y Morus, I. R. (2005). Panorama general de la ciencia moderna. Barcelona: Crítica.

Brock, W. H. (1998). Historia de la química. Madrid: Alianza. 
Caldin, E. F. (2002). The structure of chemistry in relation of the philosophy of science. International Journal for Philosophy of Chemistry, Vol. 8, No. 2, 103 121. En línea: http://hyle.org/journal/issues/8-2/caldin.htlm

Camacho Gonzáles, J. P., Gallego Badillo, R. y Pérez Miranda, R. (2007). La ley periódica. Un análisis histórico epistemológico y didáctico de algunos textos de enseñanza. Educación Química, Vol. 18, No. 4, 278 - 288.

Chevallard, I. (1985). La transposition didactique. Du savoir savant au savoir enseigné. Grenoble: La pensée Sauvage.

Cuellar Fernández, L. H., Gallego Badillo, R. y Pérez Miranda, R. (2008). El modelo atómico de E. Rutherford. Del saber científico al conocimiento escolar. Enseñanza de las Ciencias, 26(1), 43 - 52.

Del Re, G. (2000). Models and analogies in science. International Journal for Philosophy of Chemistry, Vol. 6, No. 1, 5 - 15. En línea: http://hyle.org/journal/issues/6-1/delre.htlm

Gallego Badillo, R. (1998). Discurso constructivista sobre las tecnologías. Una mirada epistemológica. Bogotá: Cooperativa Editorial Magisterio.

Gallego Badillo, R, Pérez Miranda, R. Gallego Torres, A. P. y Torres de Gallego, L. N (2006). El objeto de saber de los químicos. Formulación, modificación y abandono del modelo icónico inicial. Investigações em Ensino de Ciencias, Vol. 11, No. 3. En línea: http://www.if.ufrgs.br/ciencia/artigos/ArtigoID158/v11 n3 a2006.pdf

Gallego Badillo, R., Pérez Miranda, R. y Rincón Pabón, I. (2008). La Escuela Normal Superior de Colombia. Desde sus inicios hasta 1953. Informe final de investigación. Bogotá: Universidad Pedagógica Nacional - CIUP.

Ihde, A. J. (1984). The development of modern chemistry. New York: Dover.

Izquierdo, M. (1988). La contribución de la teoría del flogisto a la estructuración actual de la ciencia química. Implicaciones didácticas. Enseñanza de las Ciencias, $6(1), 67-74$.

Javanovic, B. (1980). Los orígenes de la minería del cobre en Europa. Investigación y Ciencia, No. 45, $94-101$.

Jensen, W. B. (1998a). Logic, history and the chemistry textbooks I: Does chemistry have a logical structure? Journal of Chemical Education, Vol. 75, No. 6, 679 687.

Jensen, W. B. (1998b). Logic, history and the chemistry textbooks II. Can we unmuddle the chemistry textbook? Journal of Chemical Education, Vol. 75, No. 7, $917-828$.

Jensen, W. B. (1998b). Logic, history and the chemistry textbooks III: One chemical revolution or tree? Journal of Chemical Education, Vol. 75, No. 8, 961 - 969.

Kant, E. (1987). Principios metafísicos de la ciencia de la naturaleza. Revista de la Universidad de Antioquia, Vol. 54, No. 209, 28-39. 
Laín Entralgo, P. y López Piñero, J. M. (1963). Panorama histórico de la ciencia moderna. Madrid: Guadarrama.

Lakatos, I. (1983). La metodología de los programas de investigación científica. Madrid: Alianza.

Laudan, R. (2005). La "nueva" historia de la ciencia: implicaciones para la filosofía de la ciencia. En: Historia, filosofía y enseñanza de la ciencia, S. F. Martínez y G. Guillaumin (Comp.), pp. 121 - 130. (Traducción de R. Bárcenas de Anda). México: UNAM.

Leidler, K. J. (1995). The world of Physical Chemistry. New York: Oxford University Press.

Levi-Strauss, C. (1964). El pensamiento salvaje. México: Fondo de Cultura Económica.

Lockemann, G. (1960). Historia de la química. México: UTEHA.

Mayr, E. (2006). Por qué es única la biología. Consideraciones sobre la autonomía de una disciplina científica. Buenos Aires: Katz.

Novak, J. D. (1982). Teoría y práctica de la educación. Madrid: Alianza.

Popper, K. (1962). La lógica de la investigación científica. Madrid: Tecnos.

Restivo, S. (1992). La ciencia moderna como problema social. Fin de Siglo, No. 3, 20 39.

Scerri, E. R. (1997). Has the periodic table successfully axiomatizad. Erkenntnis, 47, $229-343$.

Estany, A. (2005). El papel de la historia de la ciencia en los estudios interdisciplinares de la ciencia. En: Historia, filosofía y enseñanza de la ciencia, S. F. Martínez y G. Guillaumin (Comp.), pp. 291 - 303. México: UNAM.

Stengers, I. (1989). La afinidad ambigua: el sueño newtoniano de la química del siglo XVIII. En: Historia de las ciencias, M. Serres (ed.), pp. 317 - 361. Madrid: Cátedra.

Tomasi, J. (1999). Towards "chemical congruence" of the models in theoretical Chemistry. International Journal for Philosophy of Chemistry, Vol. 5, No. 2, 79 - 115. En línea: http://hyle.org/journal/issues/5-2/tomasi.htlm

Toulmin, S. (1977). La comprensión humana. Vol. 1. El uso colectivo y la evolución de los conceptos. Madrid: Alianza.

Vessuri, H. M. C. (1992). Perspectivas recientes en el estudio social de las ciencias. Fin de Siglo, No. 3, $40-52$. 


\section{THE DIDACTIC HISTORICAL CONTEXT OF THE INSTITUTIONALIZATION OF CHEMISTRY LIKE SCIENCE}

\section{SUMMARY}

They occur to know some conclusions derived from historical revisions, on like the chemistry and the work of chemistries that were constituting this science gradually and obtaining the institutionalization of her and del social recognition of its activity of knowledge production. The institutionalization is understood here, in the terms that have established the social historians of sciences, standing out the little attention that has rendered to the didactic processes for this assignment. The departure point that is adopted, is the one of which the conversion of sciences in scholastic science contributed significantly to this institutionalization. It is examined the paper of the education texts that formulated disciples and affected the creation and consolidation of a scientific community without forgetting of course, the roll fulfilled by the magazines specialized in chemistry and industrialization.

Keywords: Social history of sciences; institutionalization; texts of education. 